\title{
Refinements and Generalizations of Some Fractional Integral Inequalities via Strongly Convex Functions
}

\author{
Ghulam Farid $\mathbb{D}^{1},{ }^{1}$ Hafsa Yasmeen, ${ }^{1}$ Chahn Yong Jung $\mathbb{D}^{2},{ }^{2}$ Soo Hak Shim, ${ }^{3}$ and Gaofan Ha ${ }^{4}$ \\ ${ }^{1}$ Department of Mathematics, COMSATS University Islamabad, Attock Campus, Attock, Pakistan \\ ${ }^{2}$ Department of Business Administration, Gyeongsang National University, Jinju 52828, Republic of Korea \\ ${ }^{3}$ Department of Refrigeration and Air Conditioning Engineering, Chonnam National University, Yeosu 59626, Republic of Korea \\ ${ }^{4}$ School of Mathematics and Statistics, Northeast Normal University, Changchun 130000, China \\ Correspondence should be addressed to Chahn Yong Jung; bb5734@gnu.ac.kr
}

Received 6 November 2020; Revised 13 December 2020; Accepted 4 March 2021; Published 28 March 2021

Academic Editor: Mohammad D. Aliyu

Copyright $\odot 2021$ Ghulam Farid et al. This is an open access article distributed under the Creative Commons Attribution License, which permits unrestricted use, distribution, and reproduction in any medium, provided the original work is properly cited.

In this article, we have established the Hadamard inequalities for strongly convex functions using generalized Riemann-Liouville fractional integrals. The findings of this paper provide refinements of some fractional integral inequalities. Furthermore, the error bounds of these inequalities are given by using two generalized integral identities.

\section{Introduction}

Let $f: I \longrightarrow \mathbb{R}$ be a convex function defined on an interval $I \subset \mathbb{R}$ and $x, y \in I$, where $x<y$. Then, the following inequality holds:

$$
f\left(\frac{x+y}{2}\right) \leq \frac{1}{y-x} \int_{x}^{y} f(v) \mathrm{d} v \leq \frac{f(x)+f(y)}{2} .
$$

The above inequality is well-known as the Hadamard inequality. This inequality provides lower and upper estimates for integral average of a convex function. Since the appearance of this result in literature, it has drawn attention of many mathematicians of recent age and it is one of the most extensively studied results for convex functions. In $[1,2]$, Sarikaya et al. have studied it via Riemann-Liouville fractional integrals of convex functions. After these versions of Hadamard inequality, many researchers were motivated and elegantly produced fractional inequalities using different types of fractional integrals. Also, many new classes of functions have been introduced in the establishment of fractional Hadamard inequalities; for details, we refer the readers to [3-11].
Fractional calculus studies the integrals and derivatives of any arbitrary order, real or complex. Its history begins at the end of seventeenth century, when G. W. Leibniz and Marquis de l'Hospital in 1695 introduced it for first time by discussing the differentiation of functions of order $1 / 2$. However, it experienced a rapid growth over the short span of time. For example, Lagrange, Laplace, Lacroix, Fourier, Abel, Liouville, Riemann, Green, Holmgren, Grunwald, Letnikov, Sonin, Laurent, Nekrassov, Krug, and Weyl made their major contributions to establish a solid foundation of fractional calculus (see [12-14] and references there in). Fractional integral and derivative operators are the key factors in the development of fractional calculus. Recently, the generalizations [15-17], extensions [18-20], and applications [21-23] for fractional operators have been made by many researchers in mathematics, fluid mechanics [24-26], biological population models [27], and numerical methods [28].

Our aim in this paper is to utilize generalized Riemann-Liouville fractional integrals with monotonically increasing function. The Hadamard inequality is studied for these integral operators of strongly convex functions, and 
also, by using some integral identities, error bounds are established. Next, we give the definition of strongly convex function introduced by Polyak [29] (see also [30]).

Definition 1. Let $D$ be a convex subset of $\mathbb{X},(\mathbb{X},\|\|$.$) be a$ normed space. A function $f: D \subset \mathbb{X} \longrightarrow \mathbb{R}$ will be called strongly convex function with modulus $C \geq 0$ if

$$
f(x t+(1-t) y) \leq t f(x)+(1-t) f(y)-C t(1-t)\|y-x\|^{2},
$$

holds $\forall x, y \in D \subseteq \mathbb{X}, t \in[0,1]$. For $C=0$, (2) gives the definition of convex function.

In the following, we give the definition of Riemann-Liouville fractional integrals.

Definition 2. Let $f \in L_{1}[a, b]$. Then, left-sided and rightsided Riemann-Liouville fractional integrals of a function $f$ of order $\mu$ where $\mathfrak{R}(\mu)>0$ are defined as follows:

$$
\begin{aligned}
& I_{a^{+}}^{\mu} f(x)=\frac{1}{\Gamma(\mu)} \int_{a}^{x}(x-t)^{\mu-1} f(t) \mathrm{d} t, \quad x>a, \\
& I_{b^{-}}^{\mu} f(x)=\frac{1}{\Gamma(\mu)} \int_{x}^{b}(t-x)^{\mu-1} f(t) \mathrm{d} t, \quad x<b .
\end{aligned}
$$

The fractional versions of Hadamard inequality by Riemann-Liouville fractional integrals are given in the following theorems.

Theorem 1 (see [1]). Let $f:[a, b] \longrightarrow \mathbb{R}$ be a positive function with $0 \leq a<b$ and $f \in L_{1}[a, b]$. If $f$ is a convex function on $[a, b]$, then the following fractional integral inequalities hold:

$$
f\left(\frac{a+b}{2}\right) \leq \frac{\Gamma(\alpha+1)}{2(b-a)^{\alpha}}\left[I_{a^{+}}^{\alpha} f(b)+I_{b^{-}}^{\alpha} f(a)\right] \leq \frac{f(a)+f(b)}{2},
$$

with $\alpha>0$.

Theorem 2 (see [2]). Let $f:[a, b] \longrightarrow \mathbb{R}$ be a positive function with $0 \leq a<b$ and $f \in L_{1}[a, b]$. If $f$ is a convex function on $[a, b]$, then the following fractional integral inequalities hold:

$$
\begin{aligned}
f\left(\frac{a+b}{2}\right) & \leq \frac{2^{\alpha-1} \Gamma(\alpha+1)}{(b-a)^{\alpha}}\left[I_{(a+b / 2)^{+}}^{\alpha} f(b)+I_{(a+b / 2)^{-}}^{\alpha} f(a)\right] \\
& \leq \frac{f(a)+f(b)}{2},
\end{aligned}
$$

with $\alpha>0$.

Theorem 3 (see [1]). Let $f:[a, b] \longrightarrow \mathbb{R}$ be a differentiable mapping on $(a, b)$ with $a<b$. If $\left|f^{\prime}\right|$ is convex on $[a, b]$, then the following fractional integral inequality holds:

$$
\begin{gathered}
\left|\frac{f(a)+f(b)}{2}-\frac{\Gamma(\alpha+1)}{2(b-a)^{\alpha}}\left[I_{a^{+}}^{\alpha} f(b)+I_{b^{-}}^{\alpha} f(a)\right]\right| \\
\leq \frac{b-a}{2(\alpha+1)}\left(1-\frac{1}{2^{\alpha}}\right)\left[\left|f^{\prime}(a)\right|+\left|f^{\prime}(b)\right|\right] .
\end{gathered}
$$

In the following, refinements of Theorem 1-3 are given.

Theorem 4 (see [31]). Let $f:[a, b] \longrightarrow \mathbb{R}$ be a positive function with $0 \leq a<b$ and $f \in L_{1}[a, b]$. If $f$ is strongly convex function on $[a, b]$ with modulus $C \geq 0$, then the following fractional integral inequalities hold:

$$
\begin{gathered}
f\left(\frac{a+b}{2}\right)+\frac{C(b-a)^{2}\left(\alpha^{2}-\alpha+2\right)}{4(\alpha+1)(\alpha+2)} \\
\leq \frac{\Gamma(\alpha+1)}{2(b-a)^{\alpha}}\left[I_{a^{+}}^{\alpha} f(b)+I_{b^{-}}^{\alpha} f(a)\right] \\
\quad \leq \frac{f(a)+f(b)}{2}-\frac{C \alpha(b-a)^{2}}{(\alpha+1)(\alpha+2)}
\end{gathered}
$$

with $\alpha$.

Theorem 5 (see [32]). Let $f:[a, b] \longrightarrow \mathbb{R}$ be a positive function with $0 \leq a<b$ and $f \in L_{1}[a, b]$. If $f$ is strongly convex function on $[a, b]$ with modulus $C \geq 0$, then the following fractional integral inequalities hold:

$$
\begin{aligned}
& f\left(\frac{a+b}{2}\right)+\frac{C(b-a)^{2}}{2(\alpha+1)(\alpha+2)} \\
& \quad \leq \frac{2^{\alpha-1} \Gamma(\alpha+1)}{(b-a)^{\alpha}}\left[I_{(a+b / 2)^{+}}^{\alpha} f(b)+I_{(a+b / 2)^{-}}^{\alpha} f(a)\right] \\
& \quad \leq \frac{f(a)+f(b)}{2}-\frac{C \alpha(b-a)^{2}(\alpha+3)}{4(\alpha+1)(\alpha+2)}
\end{aligned}
$$

with $\alpha$.

Theorem 6 (see [32]). Let $f:[a, b] \longrightarrow \mathbb{R}$ be a differentiable mapping on $(a, b)$ with $a<b$ and $f \in L_{1}[a, b]$. If $f$ is strongly convex function on $[a, b]$ with modulus $C \geq 0$, then the following fractional integral inequalities hold:

$$
\begin{aligned}
& \left|\frac{f(a)+f(b)}{2}-\frac{\Gamma(\alpha+1)}{2(b-a)^{\alpha}}\left[I_{a^{+}}^{\alpha} f(b)+I_{b^{-}}^{\alpha} f(a)\right]\right| \\
& \leq \frac{b-a}{2(\alpha+1)}\left(1-\frac{1}{2^{\alpha}}\right)\left[\left|f^{\prime}(a)\right|+\left|f^{\prime}(b)\right|\right] \\
& \quad-\frac{C(b-a)^{3}}{(\alpha+2)(\alpha+3)}\left(1-\frac{\alpha+4}{2^{\alpha+2}}\right),
\end{aligned}
$$

with $\alpha$.

In [33], $k$-fractional Riemann-Liouville integrals are defined as follows. 
Definition 3. Let $f \in L_{1}[a, b]$. Then, $k$-fractional Riemann-Liouville integrals of order $\mu$, where $\mathfrak{R}(\mu)>0, k>0$, are defined by

$$
\begin{aligned}
& { }_{k} I_{a^{+}}^{\mu} f(x)=\frac{1}{k \Gamma_{k}(\mu)} \int_{a}^{x}(x-t)^{(\mu / k)-1} f(t) \mathrm{d} t, \quad x>a, \\
& { }_{k} I_{b^{-}}^{\mu} f(x)=\frac{1}{k \Gamma_{k}(\mu)} \int_{x}^{b}(t-x)^{(\mu / k)-1} f(t) \mathrm{d} t, \quad x<b,
\end{aligned}
$$

where $\Gamma_{k}($.$) is defined as follows [34]:$

$$
\Gamma_{k}(\mu)=\int_{0}^{\infty} t^{\alpha-1} e^{-\left(t^{k} / k\right)} \mathrm{d} t, \quad \mathfrak{R}(\mu)>0 .
$$

If $k=1,(11)$ and (12) coincide with (3) and (4).

Farid et al. $[35,36]$ proved the following $k$-fractional Hadamard inequalities.

Theorem 7 (see [35]). Let $f:[a, b] \longrightarrow \mathbb{R}$ be a positive function with $0 \leq a<b$. If $f$ is a convex function on $[a, b]$, then the following inequalities for $k$-fractional integrals hold:

$$
f\left(\frac{a+b}{2}\right) \leq \frac{\Gamma_{k}(\alpha+k)}{2(b-a)^{\alpha / k}}\left[{ }_{k} I_{a^{+}}^{\alpha} f(b)+{ }_{k} I_{b^{-}}^{\alpha} f(a)\right] \leq \frac{f(a)+f(b)}{2} .
$$

Theorem 8 (see [36]). Let $f:[a, b] \longrightarrow \mathbb{R}$ be a positive function with $0 \leq a<b$. If $f$ is a convex function on $[a, b]$, then the following inequalities for $k$-fractional integrals hold:

$$
\begin{aligned}
f\left(\frac{a+b}{2}\right) & \leq \frac{2^{(\alpha / k)-1} \Gamma_{k}(\alpha+k)}{(b-a)^{(\alpha / k)}}\left[{ }_{k} I_{(a+b / 2)^{+}}^{\alpha} f(b)+{ }_{k} I_{(a+b / 2)^{-}}^{\alpha} f(a)\right] \\
& \leq \frac{f(a)+f(b)}{2} .
\end{aligned}
$$

Theorem 9 (see [35]). Let $f:[a, b] \longrightarrow \mathbb{R}$ be a differentiable mapping on $(a, b)$ with $0 \leq a<b$. If $\left|f^{\prime}\right|$ is convex on $[a, b]$, then the following inequality for $k$-fractional integrals hold:

$$
\begin{gathered}
\left|\frac{f(a)+f(b)}{2}-\frac{\Gamma_{k}(\alpha+k)}{2(b-a)^{\alpha / k}}\left[{ }_{k} I_{a^{+}}^{\alpha} f(b)+{ }_{k} I_{b^{-}}^{\alpha} f(a)\right]\right| \\
\leq \frac{b-a}{2((\alpha / k)+1)}\left(1-\frac{1}{2^{(\alpha / k)}}\right)\left[\left|f^{\prime}(a)\right|+\left|f^{\prime}(b)\right|\right] .
\end{gathered}
$$

In the following, we give the definition of generalized Riemann-Liouville fractional integrals by a monotonically increasing function:
Definition 4 (see [37]). Let $f:[a, b] \longrightarrow \mathbb{R}$ be an integrable function. Also, let $\psi$ be an increasing and positive function on $(a, b]$, having a continuous derivative $\psi^{\prime}$ on $(a, b)$. The left-sided and right-sided fractional integrals of a function $f$ with respect to another function $\psi$ on $[a, b]$ of order $\mu$ where $\mathfrak{R}(\mu)>0$ are defined by

$$
I_{a^{+}}^{\mu, \psi} f(x)=\frac{1}{\Gamma(\mu)} \int_{a}^{x} \psi^{\prime}(t)(\psi(x)-\psi(t))^{\mu-1} f(t) \mathrm{d} t, \quad x>a,
$$

$$
I_{b^{-}}^{\mu, \psi} f(x)=\frac{1}{\Gamma(\mu)} \int_{x}^{b} \psi^{\prime}(t)(\psi(t)-\psi(x))^{\mu-1} f(t) \mathrm{d} t, \quad x<b
$$

If $\psi$ is identity function, then (17) and (18) coincide with (3) and (4).

The $k$-analogue of generalized Riemann-Liouville fractional integrals are defined as follows:

Definition 5 (see [38]). Let $f:[a, b] \longrightarrow \mathbb{R}$ be an integrable function. Also, let $\psi$ be an increasing and positive function on $(a, b]$, having a continuous derivative $\psi^{\prime}$ on $(a, b)$. The leftsided and right-sided fractional integrals of a function $f$ with respect to another function $\psi$ on $[a, b]$ of order $\mu$ where $\mathfrak{R}(\mu)>0, k>0$, are defined by

$$
{ }_{k} I_{a^{+}}^{\mu, \psi} f(x)=\frac{1}{k \Gamma_{k}(\mu)} \int_{a}^{x} \psi^{\prime}(t)(\psi(x)-\psi(t))^{(\mu / k)-1} f(t) \mathrm{d} t, \quad x>a,
$$

$$
{ }_{k} I_{b^{-}}^{\mu, \psi} f(x)=\frac{1}{k \Gamma_{k}(\mu)} \int_{x}^{b} \psi^{\prime}(t)(\psi(t)-\psi(x))^{(\mu / k)-1} f(t) \mathrm{d} t, \quad x<b .
$$

If $k=1,(19)$ and (20) coincide with (17) and (18). If $\psi$ is taken as identity function, (19) and (20) coincide with (11) and (12). If $\psi$ is taken as identity function along with $k=1$, (19) and (20) coincide with (3) and (4). For more details of above defined fractional integrals, one can see $[13,39]$.

In Section 2, we establish Hadamard inequalities for generalized Riemann-Liouville fractional integrals of strongly convex functions. The particular cases are given as consequences of these inequalities which are connected with already published results. In Section 3, by using two integral identities for generalized fractional integrals, the error bounds of fractional Hadamard inequalities are established. The findings of this paper are connected with results that are explicitly proved in $[1,2,31,35,36,40-44]$.

\section{Main Results}

Theorem 10. Let $f:[a, b] \longrightarrow \mathbb{R}$ be a positive function with $0 \leq a<b$ and $f \in L_{1}[a, b]$. Also, suppose that $f$ is strongly convex function on $[a, b]$ with modulus $C \geq 0, \psi$ is an increasing and positive monotone function on $(a, b]$, having $a$ 
continuous derivative $\psi^{\prime}(x)$ on $(a, b)$. Then, for $k>0$, the following $k$-fractional integral inequalities hold:

$$
\begin{aligned}
& f\left(\frac{a+b}{2}\right)+\frac{C(b-a)^{2}\left(\alpha^{2}-k \alpha+2 k^{2}\right)}{4(\alpha+k)(\alpha+2 k)} \\
& \quad \leq \frac{\Gamma_{k}(\alpha+k)}{2(b-a)^{\alpha / k}}\left[{ }_{k} I_{\psi^{-1}(a)^{+}}^{\alpha,}(f \circ \psi)\left(\psi^{-1}(b)\right)+{ }_{k} I_{\psi^{-1}(b)^{-}}^{\alpha, \psi}(f \circ \psi)\left(\psi^{-1}(a)\right)\right] \\
& \quad \leq \frac{f(a)+f(b)}{2}-\frac{C k \alpha(b-a)^{2}}{(\alpha+k)(\alpha+2 k)},
\end{aligned}
$$

with $\alpha>0$.

Proof. Since the function $f$ is strongly convex function, so for $x, y \in[a, b]$, we have

$$
f\left(\frac{x+y}{2}\right) \leq \frac{f(x)+f(y)}{2}-\frac{C}{4}|x-y|^{2} .
$$

Let $x=a t+(1-t) b, y=(1-t) a+t b$ for $t \in[0,1]$ in (22) and multiplying the resulting inequality with $t^{(\alpha / k)-1}$ on both sides, we get

$$
\begin{aligned}
2 f\left(\frac{a+b}{2}\right) t^{(\alpha / k)-1} \leq & f(a t+(1-t) b) t^{(\alpha / k)-1} \\
& +f((1-t) a+b t) t^{(\alpha / k)-1} \\
& -\frac{C}{2}(b-a)^{2}(1-2 t)^{2} t^{(\alpha / k)-1} .
\end{aligned}
$$

Integrating (23) over the interval $[0,1]$, we get

$$
\begin{aligned}
2 f\left(\frac{a+b}{2}\right) \int_{0}^{1} t^{(\alpha / k)-1} \mathrm{~d} t \leq & \int_{0}^{1} f(a t+(1-t) b) t^{(\alpha / k)-1} \mathrm{~d} t \\
& +\int_{0}^{1} f((1-t) a+b t) t^{(\alpha / k)-1} \mathrm{~d} t \\
& -\frac{C}{2}(b-a)^{2} \int_{0}^{1}(1-2 t)^{2} t^{(\alpha / k)-1} \mathrm{~d} t, \\
\frac{2 k}{\alpha} f\left(\frac{a+b}{2}\right) \leq & \int_{0}^{1} f(a t+(1-t) b) t^{(\alpha / k)-1} \mathrm{~d} t \\
& +\int_{0}^{1} f((1-t) a+b t) t^{(\alpha / k)-1} \mathrm{~d} t \\
& -\frac{C k(b-a)^{2}\left(\alpha^{2}-k \alpha+2 k^{2}\right)}{2 \alpha(\alpha+k)(\alpha+2 k)} .
\end{aligned}
$$

Multiplying (24) by $\alpha / 2 k$, we get

$$
\begin{aligned}
& f\left(\frac{a+b}{2}\right) \leq \frac{\alpha}{2 k}\left(\int_{0}^{1} f(a t+(1-t) b) t^{(\alpha / k)-1} \mathrm{~d} t\right. \\
& \left.\quad+\int_{0}^{1} f((1-t) a+b t) t^{(\alpha / k)-1} \mathrm{~d} t\right)-\frac{C(b-a)^{2}\left(\alpha^{2}-k \alpha+2 k^{2}\right)}{4(\alpha+k)(\alpha+2 k)} .
\end{aligned}
$$

Taking $u \in[a, b]$ so that $\psi(u)=a t+b(1-t)$, that is, $t=(b-\psi(u)) /(b-a)$, and $v \in[a, b]$ so that $\psi(v)=a(1-t)+b t$, that is, $t=(\psi(v)-a) /(b-a)$, in (25), then by applying Definition 5 , we get the following inequality:

$$
\begin{aligned}
& f\left(\frac{a+b}{2}\right)+\frac{C(b-a)^{2}\left(\alpha^{2}-k \alpha+2 k^{2}\right)}{4(\alpha+k)(\alpha+2 k)} \\
& \quad \leq \frac{\Gamma_{k}(\alpha+k)}{2(b-a)^{\alpha / k}}\left[{ }_{k} I_{\psi^{-1}(a)^{+}}^{\alpha, \psi}(f \circ \psi)\left(\psi^{-1}(b)\right)+{ }_{k} I_{\psi^{-1}(b)^{-}}^{\alpha, \psi}(f \circ \psi)\left(\psi^{-1}(a)\right)\right] .
\end{aligned}
$$

Since $f$ is strongly convex function, for $t \in[0,1]$, we also have following inequality:

$$
\begin{aligned}
f(t a+(1-t) b)+f((1-t) a+t b) \leq & f(a)+f(b) \\
& -2 C t(1-t)(b-a)^{2} .
\end{aligned}
$$

Multiplying (27) with $t^{(\alpha / k)-1}$ and then integrating over the interval $[0,1]$, we get

$$
\begin{aligned}
& \int_{0}^{1} t^{(\alpha / k)-1} f(t a+(1-t) b) \mathrm{d} t+\int_{0}^{1} t^{(\alpha / k)-1} f((1-t) a+t b) \mathrm{d} t \\
& \leq(f(a)+f(b)) \int_{0}^{1} t^{(\alpha / k)-1} \mathrm{~d} t-2 C(b-a)^{2} \int_{0}^{1} t^{(\alpha / k)}(1-t) \mathrm{d} t \\
& \int_{0}^{1} t^{(\alpha / k)-1} f(t a+(1-t) b) \mathrm{d} t+\int_{0}^{1} t^{(\alpha / k)-1} f((1-t) a+t b) \mathrm{d} t \\
& \leq \frac{k(f(a)+f(b))}{\alpha}-\frac{2 C k^{2}(b-a)^{2}}{(\alpha+k)(\alpha+2 k)}
\end{aligned}
$$

Multiplying (28) by $\alpha / 2 k$, we get

$$
\begin{aligned}
& \frac{\alpha}{2 k}\left(\int_{0}^{1} t^{(\alpha / k)-1} f(t a+(1-t) b) \mathrm{d} t+\int_{0}^{1} t^{(\alpha / k)-1} f((1-t) a+t b) \mathrm{d} t\right) \\
& \quad \leq \frac{f(a)+f(b)}{2}-\frac{C k \alpha(b-a)^{2}}{(\alpha+k)(\alpha+2 k)} .
\end{aligned}
$$

Again taking $\psi(u)=a t+b(1-t)$, that is, $t=(b-\psi(u) / b-a)$, and $\psi(v)=a(1-t)+b t$, that is, $t=(\psi(v)-a /(b-a))$, in (29), then by applying Definition 5 , we get the following inequality:

$$
\begin{aligned}
& \frac{\Gamma_{k}(\alpha+k)}{2(b-a)^{\alpha / k}}\left[{ }_{k} I_{\psi^{-1}(a)^{+}}^{\alpha, \psi}(f \circ \psi)\left(\psi^{-1}(b)\right)+{ }_{k} I_{\psi^{-1}(b)^{-}}^{\alpha, \psi}(f \circ \psi)\left(\psi^{-1}(a)\right)\right] \\
& \quad \leq \frac{f(a)+f(b)}{2}-\frac{C k \alpha(b-a)^{2}}{(\alpha+k)(\alpha+2 k)} .
\end{aligned}
$$

Combining (26) and (30), we get (21).

Remark 1. Under the assumption of Theorem 10, one can get the following results: 
(i) If $C=0, k=1$, and $\psi$ is identity function in (21), then Theorem 1 is obtained.

(ii) If $C=0$ and $\psi$ is identity function in (21), then Theorem 7 is obtained.

(iii) If $k=1$ and $\psi$ is identity function in (21), then Theorem 4 is obtained.

(iv) If $\alpha=1, k=1, C=0$, and $\psi$ is identity function in (21), then Hadamard inequality is obtained.

(v) If $C=0$ in (21), then the inequality (Theorem 1) stated in [41] is obtained.

(vi) If $C=0$ and $k=1$ in (21), then the inequality (Theorem 2.1) stated in [40] is obtained.

(vii) If $k=1, \alpha=1$, and $\psi$ is identity function in (21), then the inequality (Theorem 6) stated in [44] is obtained.

Corollary 1. Under the assumption of Theorem 10 with $k=1$ in (21), the following inequality holds:

$$
\begin{aligned}
& f\left(\frac{a+b}{2}\right)+\frac{C(b-a)^{2}\left(\alpha^{2}-\alpha+2\right)}{4(\alpha+1)(\alpha+2)} \\
& \quad \leq \frac{\Gamma(\alpha+1)}{2(b-a)^{\alpha}}\left[I_{\psi^{-1}(a)^{+}}^{\alpha, \psi}(f \circ \psi)\left(\psi^{-1}(b)\right)+I_{\psi^{-1}(b)^{-}}^{\alpha, \psi}(f \circ \psi)\left(\psi^{-1}(a)\right)\right] \\
& \quad \leq \frac{f(a)+f(b)}{2}-\frac{C \alpha(b-a)^{2}}{(\alpha+1)(\alpha+2)} .
\end{aligned}
$$

Corollary 2. Under the assumption of Theorem 10 with $\psi$ as identity function in (21), the following inequality holds:

$$
\begin{gathered}
f\left(\frac{a+b}{2}\right)+\frac{C(b-a)^{2}\left(\alpha^{2}-k \alpha+2 k^{2}\right)}{4(\alpha+k)(\alpha+2 k)} \\
\quad \leq \frac{\Gamma_{k}(\alpha+k)}{2(b-a)^{\alpha / k}}\left[{ }_{k} I_{a^{+}}^{\alpha} f(b)+{ }_{k} I_{b^{-}}^{\alpha} f(a)\right] \\
\quad \leq \frac{f(a)+f(b)}{2}-\frac{C k \alpha(b-a)^{2}}{(\alpha+k)(\alpha+2 k)} .
\end{gathered}
$$

Theorem 11. Let $f:[a, b] \longrightarrow \mathbb{R}$ be a positive function with $0 \leq a<b$ and $f \in L_{1}[a, b]$. Also, suppose that $f$ is strongly convex function on $[a, b]$ with modulus $C \geq 0$ and $\psi$ is an increasing and positive monotone function on $(a, b]$, having $a$ continuous derivative $\psi^{\prime}(x)$ on $(a, b)$. Then, for $k>0$, the following $k$-fractional integral inequalities hold:

$$
\begin{aligned}
& f\left(\frac{a+b}{2}\right)+\frac{k^{2} C(b-a)^{2}}{2(\alpha+k)(\alpha+2 k)} \\
& \quad \leq \frac{2^{(\alpha / k)-1} \Gamma_{k}(\alpha+k)}{(b-a)^{(\alpha / k)}}\left[{ }_{k} I_{\psi^{-1}(a+b / 2)^{+}}^{\alpha, \psi}(f \circ \psi)\left(\psi^{-1}(b)\right)+{ }_{k} I_{\psi^{-1}(a+b / 2)^{-}}^{\alpha, \psi}(f \circ \psi)\left(\psi^{-1}(a)\right)\right] \\
& \quad \leq \frac{f(a)+f(b)}{2}-\frac{C \alpha(b-a)^{2}(\alpha+3 k)}{4(\alpha+k)(\alpha+2 k)},
\end{aligned}
$$

with $\alpha>0$.

Proof. Let $x=(a t / 2)+(2-t / 2) b$ and $y=(2-t / 2) a+$ $(b t / 2)$ in (22), and multiplying the resulting inequality with $t^{(\alpha / k)-1}$, we get

$$
\begin{aligned}
f\left(\frac{a+b}{2}\right) t^{(\alpha / k)-1} \leq & \frac{1}{2}\left[f\left(\frac{a t}{2}+\left(\frac{2-t}{2}\right) b\right) t^{(\alpha / k)-1}+f\left(\left(\frac{2-t}{2}\right) a+\frac{b t}{2}\right) t^{(\alpha / k)-1}\right] \\
& -\frac{C}{4}(b-a)^{2}(1-t)^{2} t^{(\alpha / k)-1}
\end{aligned}
$$


Integrating (34) over $[0,1]$, we get

$$
\frac{2 k}{\alpha} f\left(\frac{a+b}{2}\right) \leq \int_{0}^{1} f\left(\frac{a t}{2}+\left(\frac{2-t}{2}\right) b\right) t^{(\alpha / k)-1} \mathrm{~d} t+\int_{0}^{1} f\left(a\left(\frac{2-t}{2}\right)+\frac{b t}{2}\right) t^{(\alpha / k)-1} \mathrm{~d} t-\frac{k^{3} C(b-a)^{2}}{\alpha(\alpha+k)(\alpha+2 k)}
$$

Multiplying above inequality with $\alpha / 2 k$, we get

$$
f\left(\frac{a+b}{2}\right)+\frac{C k^{2}(b-a)^{2}}{2(\alpha+k)(\alpha+2 k)} \leq \frac{\alpha}{2 k}\left(\int_{0}^{1} f\left(\frac{a t}{2}+\left(\frac{2-t}{2}\right) b\right) t^{(\alpha / k)-1} \mathrm{~d} t+\int_{0}^{1} f\left(a\left(\frac{2-t}{2}\right)+\frac{b t}{2}\right) t^{(\alpha / k)-1} \mathrm{~d} t\right) .
$$

Taking $u \in[a, b]$ so that $\psi(u)=(a t / 2)+b(2-t / 2)$, that is, $t=(2(b-\psi(u)) / b-a)$, and $v \in[a, b]$ so that $\psi(v)=a(2-t / 2)+(b t / 2)$, that is, $t=(2(\psi(v)-a) / b-a)$,

in (36), then by applying Definition 5, we get following inequality:

$$
f\left(\frac{a+b}{2}\right)+\frac{C k^{2}(b-a)^{2}}{2(\alpha+k)(\alpha+2 k)} \leq \frac{2^{(\alpha / k)-1} \Gamma_{k}(\alpha+k)}{(b-a)^{(\alpha / k)}}\left[{ }_{k} I_{\psi^{-1}(a+b / 2)^{+}}^{\alpha, \psi}(f \circ \psi)\left(\psi^{-1}(b)\right)+{ }_{k} I_{\psi^{-1}(a+b / 2)^{-}}^{\alpha, \psi}(f \circ \psi)\left(\psi^{-1}(a)\right)\right] .
$$

Since $f$ is strongly convex function on $[a, b]$, for $t \in[0,1]$, we have the following inequality:

$$
f\left(\frac{a t}{2}+\left(\frac{2-t}{2}\right) b\right)+f\left(a\left(\frac{2-t}{2}\right)+\frac{b t}{2}\right) \leq f(a)+f(b)-\frac{C t(2-t)(b-a)^{2}}{2}
$$

Multiplying (38) with $t^{(\alpha / k)-1}$ on both sides and integrating over $[0,1]$, we get

$$
\begin{gathered}
\int_{0}^{1} f\left(\frac{a t}{2}+\left(\frac{2-t}{2}\right) b\right) t^{(\alpha / k)-1} \mathrm{~d} t+\int_{0}^{1} f\left(a\left(\frac{2-t}{2}\right)+\frac{b t}{2}\right) t^{(\alpha / k)-1} \mathrm{~d} t \\
\quad \leq(f(a)+f(b)) \int_{0}^{1} t^{(\alpha / k)-1} \mathrm{~d} t-\frac{C(b-a)^{2}}{2} \int_{0}^{1}(2-t) t^{(\alpha / k)} \mathrm{d} t .
\end{gathered}
$$

Multiplying (39) with $\alpha / 2 k$ on both sides, we get

$$
\begin{aligned}
& \frac{\alpha}{2 k}\left(\int_{0}^{1} f\left(\frac{a t}{2}+\left(\frac{2-t}{2}\right) b\right) t^{(\alpha / k)-1} \mathrm{~d} t+\int_{0}^{1} f\left(\left(\frac{2-t}{2}\right) a+\frac{b t}{2}\right) t^{(\alpha / k)-1} \mathrm{~d} t\right) \\
& \quad \leq \frac{f(a)+f(b)}{2}-\frac{C \alpha(b-a)^{2}(\alpha+3 k)}{4(\alpha+k)(\alpha+2 k)} .
\end{aligned}
$$

Again taking $\psi(u)=(a t / 2)+b(2-t / 2)$, that is, $t=(2(b-\psi(v)) / b-a)$, and $\psi(v)=a(2-t / 2)+(b t / 2)$, that is, $t=(2(\psi(v)-a) / b-a)$, in (40), then by applying Definition 5 , we get the following inequality:

$$
\begin{aligned}
& \frac{2^{\alpha / k} \Gamma_{k}(\alpha+k)}{2(b-a)^{\alpha / k}}\left[{ }_{k} I_{\psi^{-1}(a+b / 2)^{+}}^{\alpha, \psi}(f \circ \psi)\left(\psi^{-1}(b)\right)+{ }_{k} I_{\psi^{-1}(a+b / 2)^{-}}^{\alpha, \psi}(f \circ \psi)\left(\psi^{-1}(a)\right)\right] \\
& \quad \leq \frac{f(a)+f(b)}{2}-\frac{C \alpha(b-a)^{2}(\alpha+3 k)}{4(\alpha+k)(\alpha+2 k)} .
\end{aligned}
$$


Combining (37) and (41), (33) is obtained.

Remark 2. Under the assumption of Theorem 11, one can get the following results:

(i) If $k=1, C=0$, and $\psi$ is identity function in (33), then Theorem 2 is obtained.

(ii) If $k=1$ and $\psi$ is identity function in (33), then Theorem 5 is obtained.

(iii) If $C=0$ and $\psi$ is identity function in (33), then inequality (Theorem 2.1) stated in [36] is obtained. (iv) If $k=1, C=0, \alpha=1$, and $\psi$ is identity function in (33), then Hadamard inequality is obtained.

(v) If $k=1, \alpha=1$, and $\psi$ is identity function in (33), then the inequality (Theorem 6) stated in [44] is obtained.

Corollary 3. Under the assumption of Theorem 11 with $C=$ 0 in (33), the following inequality holds:

$$
\begin{aligned}
f\left(\frac{a+b}{2}\right) & \leq \frac{2^{(\alpha / k)-1} \Gamma_{k}(\alpha+k)}{(b-a)^{(\alpha / k)}}\left[{ }_{k} I_{\psi^{-1}(a+b / 2)^{+}}^{\alpha, \psi}(f \circ \psi)\left(\psi^{-1}(b)\right)+{ }_{k} I_{\psi^{-1}(a+b / 2)^{-}}^{\alpha, \psi}(f \circ \psi)\left(\psi^{-1}(a)\right)\right] \\
& \leq \frac{f(a)+f(b)}{2} .
\end{aligned}
$$

Corollary 4. Under the assumption of Theorem 11 with $k=1$

in (33), the following inequality holds:

$$
\begin{aligned}
f\left(\frac{a+b}{2}\right)+\frac{C(b-a)^{2}}{2(\alpha+1)(\alpha+2)} & \leq \frac{2^{\alpha-1} \Gamma(\alpha+1)}{(b-a)^{\alpha}}\left[I_{\psi^{-1}(a+b / 2)^{+}}^{\alpha, \psi}(f \circ \psi)\left(\psi^{-1}(b)\right)+I_{\psi^{-1}(a+b / 2)^{-}}^{\alpha, \psi}(f \circ \psi)\left(\psi^{-1}(a)\right)\right] \\
& \leq \frac{f(a)+f(b)}{2}-\frac{C \alpha(b-a)^{2}(\alpha+3)}{4(\alpha+1)(\alpha+2)} .
\end{aligned}
$$

Corollary 5. Under the assumption of Theorem 11 with $\psi$ as identity function in (33), the following inequality holds:

$$
\begin{aligned}
& f\left(\frac{a+b}{2}\right)+\frac{C k^{2}(b-a)^{2}}{2(\alpha+k)(\alpha+2 k)} \\
& \quad \leq \frac{2^{(\alpha / k)-1} \Gamma_{k}(\alpha+k)}{(b-a)^{(\alpha / k)}}\left[{ }_{k} I_{(a+b / 2)^{+}}^{\alpha} f(b)+{ }_{k} I_{(a+b / 2)^{-}}^{\alpha} f(a)\right] \\
& \quad \leq \frac{f(a)+f(b)}{2}-\frac{C \alpha(b-a)^{2}(\alpha+3 k)}{4(\alpha+k)(\alpha+2 k)} .
\end{aligned}
$$

\section{Error Bounds of Hadamard Inequalities for Strongly Convex Functions}

In this section, we provide the error bounds of fractional Hadamard inequalities using generalized Riemann-Liouville fractional integrals via strongly convex functions. Estimations here are further refined as compared to those already established for convex functions. The following lemma is useful to prove the next result.

Lemma 1 (see [41]). Let $a<b$ and $f:[a, b] \longrightarrow \mathbb{R}$ be $a$ differentiable mapping on $(a, b)$. Also, suppose that $f^{\prime} \in L[a, b], \psi$ is an increasing and positive monotone function on $(a, b]$, having a continuous derivative $\psi^{\prime}(x)$ on $(a, b)$, and $\alpha \in(0,1)$. Then, for $k>0$, the following identity holds:

$$
\begin{aligned}
& \frac{f(a)+f(b)}{2}-\frac{\Gamma_{k}(\alpha+k)}{2(b-a)^{\alpha / k}}\left[{ }_{k} I_{\psi^{-1}(a)^{+}}^{\alpha,}(f \circ \psi)\left(\psi^{-1}(b)\right)+{ }_{k} I_{\psi^{-1}(b)^{-}}^{\alpha,}(f \circ \psi)\left(\psi^{-1}(a)\right)\right] \\
& =\frac{b-a}{2} \int_{0}^{1}\left[(1-t)^{\alpha / k}-t^{\alpha / k}\right] f^{\prime}(t a+(1-t) b) \mathrm{d} t .
\end{aligned}
$$


Theorem 12. Let $f:[a, b] \longrightarrow \mathbb{R}$ be a differentiable mapping on $(a, b)$ with $a<b$. Also, suppose that $\left|f^{\prime}\right|$ is strongly convex function on $[a, b]$ with modulus $C \geq 0$ and $\psi(x)$ is an increasing and positive monotone function on $(a, b]$, having a continuous derivative $\psi^{\prime}(x)$ on $(a, b)$. Then, for $k>0$, the following $k$-fractional integral inequalities hold:

$$
\begin{aligned}
& \left|\frac{f(a)+f(b)}{2}-\frac{\Gamma_{k}(\alpha+k)}{2(b-a)^{\alpha / k}}\left[{ }_{k} I_{\psi^{-1}(a)^{+}}^{\alpha, \psi}(f \circ \psi)\left(\psi^{-1}(b)\right)+{ }_{k} I_{\psi^{-1}(b)^{-}}^{\alpha, \psi}(f \circ \psi)\left(\psi^{-1}(a)\right)\right]\right| \\
& \quad \leq \frac{b-a}{2((\alpha / k)+1)}\left(1-\frac{1}{2^{(\alpha / k)}}\right)\left[\left|f^{\prime}(a)\right|+\left|f^{\prime}(b)\right|\right]-\frac{C(b-a)^{3}}{((\alpha / k)+2)((\alpha / k)+3)}\left(1-\frac{(\alpha / k)+4}{2^{(\alpha / k)+2}}\right),
\end{aligned}
$$

with $\alpha>0$.

Proof. From Lemma 1 and strongly convexity of $\left|f^{\prime}\right|$, we have

$$
\begin{aligned}
& \left|\frac{f(a)+f(b)}{2}-\frac{\Gamma_{k}(\alpha+k)}{2(b-a)^{(\alpha / k)}}\left[{ }_{k} I_{\psi^{-1}(a)^{+}}^{\alpha, \psi}(f \circ \psi)\left(\psi^{-1}(b)\right)+{ }_{k} I_{\psi^{-1}(b)^{-}}^{\alpha}(f \circ \psi)\left(\psi^{-1}(b)\right)\right]\right| \\
& \quad \leq \frac{b-a}{2} \int_{0}^{1}\left|(1-t)^{(\alpha / k)}-t^{(\alpha / k)}\right|\left|f^{\prime}(t a+(1-t))\right| \mathrm{d} t \\
& \quad \leq \frac{b-a}{2} \int_{0}^{1}\left|(1-t)^{(\alpha / k)}-t^{(\alpha / k)}\right|\left(t\left|f^{\prime}(a)\right|+(1-t)\left|f^{\prime}(b)\right|-C t(1-t)|b-a|^{2}\right) \mathrm{d} t \\
& \quad=\frac{b-a}{2} \int_{0}^{1 / 2}\left((1-t)^{(\alpha / k)}-t^{(\alpha / k)}\right)\left(t\left|f^{\prime}(a)\right|+(1-t)\left|f^{\prime}(b)\right|-C t(1-t)|b-a|^{2}\right) \mathrm{d} t \\
& \quad+\int_{1 / 2}^{1}\left(t^{(\alpha / k)}-(1-t)^{(\alpha / k)}\right)\left(t\left|f^{\prime}(a)\right|+(1-t)\left|f^{\prime}(b)\right|-C t(1-t)|b-a|^{2}\right) \mathrm{d} t .
\end{aligned}
$$

It can be noted that

$$
\begin{aligned}
& \int_{0}^{1 / 2}\left((1-t)^{\alpha / k}-t^{\alpha / k}\right)\left(t\left|f^{\prime}(a)\right|+(1-t)\left|f^{\prime}(b)\right|-C t(1-t)|b-a|^{2}\right) \mathrm{d} t \\
& =\left|f^{\prime}(a)\right| \int_{0}^{1 / 2}\left(t(1-t)^{\alpha / k}-t^{(\alpha / k)+1}\right) \mathrm{d} t+\left|f^{\prime}(b)\right| \int_{0}^{1 / 2}\left((1-t)^{(\alpha / k)+1}-(1-t) t^{\alpha / k}\right) \mathrm{d} t \\
& \quad-C(b-a)^{2}\left(\int_{0}^{1 / 2} t(1-t)^{(\alpha / k)+1} \mathrm{~d} t-\int_{0}^{1 / 2}(1-t) t^{(\alpha / k)+1} \mathrm{~d} t\right) \\
& =\left|f^{\prime}(a)\right|\left(\frac{1}{((\alpha / k)+1)((\alpha / k)+2)}-\frac{(1 / 2)^{(\alpha / k)+1}}{(\alpha / k)+1}\right)+\left|f^{\prime}(b)\right|\left(\frac{1}{(\alpha / k)+2}-\frac{(1 / 2)^{(\alpha / k)+1}}{(\alpha / k)+1}\right) \\
& \quad-\frac{C(b-a)^{2}}{((\alpha / k)+2)((\alpha / k)+3)}\left(1-\frac{(\alpha / k)+4}{2^{(\alpha / k)+2}}\right) .
\end{aligned}
$$


By similar evaluation, one can have

$$
\begin{aligned}
\int_{1 / 2}^{1} & \left(t^{(\alpha / k)}-(1-t)^{(\alpha / k)}\right)\left(t\left|f^{\prime}(a)\right|+(1-t)\left|f^{\prime}(b)\right|-C t(1-t)|b-a|^{2}\right) \mathrm{d} t \\
= & \left|f^{\prime}(a)\right|\left(\frac{1}{(\alpha / k)+2}-\frac{(1 / 2)^{(\alpha / k)+1}}{(\alpha / k)+1}\right)+\left|f^{\prime}(b)\right|\left(\frac{1}{((\alpha / k)+1)((\alpha / k)+2)}-\frac{(1 / 2)^{(\alpha / k)+1}}{(\alpha / k)+1}\right) \\
& -\frac{C(b-a)^{2}}{((\alpha / k)+2)((\alpha / k)+3)}\left(1-\frac{(\alpha / k)+4}{2^{(\alpha / k)+2}}\right) .
\end{aligned}
$$

Therefore, (47) implies

$$
\begin{aligned}
& \left|\frac{f(a)+f(b)}{2}-\frac{\Gamma_{k}(\alpha+k)}{2(b-a)^{(\alpha / k)}}\left[k_{\psi^{-1}(a)^{+}}^{\alpha, \psi}(f \circ \psi)\left(\psi^{-1}(b)\right)+{ }_{k} I_{\psi^{-1}(b)^{-}}^{\alpha}(f \circ \psi)\left(\psi^{-1}(b)\right)\right]\right| \\
& \leq \frac{b-a}{2}\left[\left|f^{\prime}(a)\right|\left(\frac{1}{((\alpha / k)+1)((\alpha / k)+2)}-\frac{(1 / 2)^{(\alpha / k)+1}}{(\alpha / k)+1}\right)+\left|f^{\prime}(b)\right|\left(\frac{1}{(\alpha / k)+2}-\frac{(1 / 2)^{(\alpha / k)+1}}{(\alpha / k)+1}\right)\right. \\
& \quad-\frac{C(b-a)^{2}}{((\alpha / k)+2)((\alpha / k)+3)}\left(1-\frac{(\alpha / k)+4}{2^{(\alpha / k)+2}}\right)+\left|f^{\prime}(a)\right|\left(\frac{1}{(\alpha / k)+2}-\frac{(1 / 2)^{(\alpha / k)+1}}{(\alpha / k)+1}\right) \\
& \left.\quad+\left|f^{\prime}(b)\right|\left(\frac{1}{((\alpha / k)+1)((\alpha / k)+2)}-\frac{(1 / 2)^{(\alpha / k)+1}}{(\alpha / k)+1}\right)-\frac{C(b-a)^{2}}{((\alpha / k)+2)((\alpha / k)+3)}\left(1-\frac{(\alpha / k)+4}{2^{(\alpha / k)+2}}\right)\right] .
\end{aligned}
$$
(46).

From which after a little computation, one can get

Remark 3. Under the assumption of Theorem 12, one can get the following results:

(i) If $k=1$ and $\psi$ is identity function in (46), then Theorem 6 is obtained.

(ii) If $C=0$ and $\psi$ is identity function in (46), then Theorem 9 is obtained. (iii) If $k=1, C=0$, and $\psi$ is identity function in (46), then Theorem 3 is obtained.

(iv) If $k=1, C=0, \alpha=1$, and $\psi$ is identity function in (46), then Theorem 2.2 in [42] is obtained.

(v) If $k=1$ and $\psi$ is identity function in (46), then Theorem 6 is obtained.

Corollary 6. Under the assumption of Theorem 12 with $C=$ 0 in (46), the following inequality holds:

$$
\begin{aligned}
& \left|\frac{f(a)+f(b)}{2}-\frac{\Gamma_{k}(\alpha+k)}{2(b-a)^{(\alpha / k)}}\left[{ }_{k} I_{\psi^{-1}(a)^{+}}^{\alpha, \psi}(f \circ \psi)\left(\psi^{-1}(b)\right)+{ }_{k} I_{\psi^{-1}(b)^{-}}^{\alpha, \psi}(f \circ \psi)\left(\psi^{-1}(a)\right)\right]\right| \\
& \quad \leq \frac{b-a}{2((\alpha / k)+1)}\left(1-\frac{1}{2^{(\alpha / k)}}\right)\left[\left|f^{\prime}(a)\right|+\left|f^{\prime}(b)\right|\right] .
\end{aligned}
$$

Corollary 7. Under the assumption of Theorem 12 with $k=1$

in (46), the following inequality holds:

$$
\begin{aligned}
& \left|\frac{f(a)+f(b)}{2}-\frac{\Gamma(\alpha+1)}{2(b-a)^{\alpha}}\left[I_{\psi^{-1}(a)^{+}}^{\alpha, \psi}(f \circ \psi)\left(\psi^{-1}(b)\right)+I_{\psi^{-1}(b)^{-}}^{\alpha,}(f \circ \psi)\left(\psi^{-1}(a)\right)\right]\right| \\
& \leq \frac{b-a}{2(\alpha+1)}\left(1-\frac{1}{2^{\alpha}}\right)\left[\left|f^{\prime}(a)\right|+\left|f^{\prime}(b)\right|\right]-\frac{C(b-a)^{3}}{(\alpha+2)(\alpha+3)}\left(1-\frac{\alpha+4}{2^{\alpha+2}}\right) .
\end{aligned}
$$


Corollary 8. Under the assumption of Theorem 12 with $\psi$ as identity function in (46), the following inequality holds:

$$
\begin{aligned}
& \left|\frac{f(a)+f(b)}{2}-\frac{\Gamma_{k}(\alpha+k)}{2(b-a)^{\alpha / k}}\left[{ }_{k} I_{a^{+}}^{\alpha} f(b)+{ }_{k} I_{b^{-}}^{\alpha} f(a)\right]\right| \\
& \quad \leq \frac{b-a}{2((\alpha / k)+1)}\left(1-\frac{1}{2^{\alpha / k}}\right)\left[\left|f^{\prime}(a)\right|+\left|f^{\prime}(b)\right|\right]-\frac{C(b-a)^{3}}{((\alpha / k)+2)((\alpha / k)+3)}\left(1-\frac{(\alpha / k)+4}{2^{(\alpha / k)+2}}\right) .
\end{aligned}
$$

We now derive a new fractional integral identity for fractional integrals (19) and (20).

Lemma 2. Let $a<b$ and $f:[a, b] \longrightarrow \mathbb{R}$ be a differentiable mapping on $(a, b)$. Also, suppose that $f^{\prime} \in L[a, b]$ and $\psi$ is an increasing and positive monotone function on $(a, b]$, having $a$ continuous derivative $\psi^{\prime}(x)$ on $(a, b)$. Then, for $k>0$, the following identity holds:

$$
\begin{aligned}
& \frac{2^{(\alpha / k)-1} \Gamma_{k}(\alpha+k)}{(b-a)^{(\alpha / k)}}\left[{ }_{k} I_{\psi^{-1}(a+b / 2)^{+}}^{\alpha, \psi}(f \circ \psi)\left(\psi^{-1}(b)\right)+{ }_{k} I_{\psi^{-1}(a+b / 2)^{-}}^{\alpha, \psi}(f \circ \psi)\left(\psi^{-1}(a)\right)\right]-f\left(\frac{a+b}{2}\right) \\
& \quad=\frac{b-a}{4}\left[\int_{0}^{1} t^{(\alpha / k)} f^{\prime}\left(\frac{a t}{2}+\left(\frac{2-t}{2}\right) b\right) \mathrm{d} t\right. \\
& \left.\quad-\int_{0}^{1} t^{(\alpha / k)} f^{\prime}\left(\left(\frac{2-t}{2}\right) a+\frac{b t}{2}\right) \mathrm{d} t\right],
\end{aligned}
$$

with $\alpha>0$.

Then, we have

Proof. Let

$$
\begin{aligned}
& I_{1}=\frac{2^{(\alpha / k)-1} \Gamma_{k}(\alpha+k)}{(b-a)^{(\alpha / k)}}\left[{ }_{k} I_{\psi^{-1}(a+b / 2)^{+}}^{\alpha, \psi}(f \circ \psi)\left(\psi^{-1}(b)\right)\right], \\
& I_{2}=\frac{2^{(\alpha / k)-1} \Gamma_{k}(\alpha+k)}{(b-a)^{(\alpha / k)}}\left[{ }_{k} I_{\psi^{-1}(a+b / 2)^{-}}^{\alpha, \psi}(f \circ \psi)\left(\psi^{-1}(a)\right)\right] .
\end{aligned}
$$

$$
\begin{aligned}
I_{1} & \left.=\frac{2^{(\alpha / k)-1} \alpha}{k(b-a)^{(\alpha / k)}}\left[\int_{\psi^{-1}(a+b / 2)}^{\psi^{-1}(b)} \psi^{\prime}(v)(b-\psi(v))^{(\alpha / k)-1}(f \circ \psi)(v)\right) \mathrm{d} v\right] \\
& =\frac{-2^{(\alpha / k)-1}}{(b-a)^{(\alpha / k)}}\left[\int_{\psi^{-1}(a+b / 2)}^{\psi^{-1}(b)} f(\psi(v)) d(b-\psi(v))^{(\alpha / k)}\right] \\
& =\frac{1}{2} f\left(\frac{a+b}{2}\right)+\frac{1}{2} \int_{\psi^{-1}(a+b / 2)}^{\psi^{-1}(b)} f^{\prime}(\psi(v))\left(\frac{2(b-\psi(v))}{b-a}\right)^{(\alpha / k)} \psi^{\prime}(v) \mathrm{d} v .
\end{aligned}
$$

By substituting $t=(2(b-\psi(v)) / b-a)$, we will get 


$$
I_{1}=\frac{1}{2} f\left(\frac{a+b}{2}\right)+\frac{b-a}{4} \int_{0}^{1} t^{\alpha / k} f^{\prime}\left(\frac{a t}{2}+\left(\frac{2-t}{2}\right) b\right) \mathrm{d} t
$$

We also have

$$
\begin{aligned}
I_{2} & =\frac{2^{(\alpha / k)-1} \alpha}{k(b-a)^{(\alpha / k)}}\left[\int_{\psi^{-1}(a)}^{\psi^{-1}(a+b / 2)} \psi^{\prime}(v)(\psi(v)-a)^{(\alpha / k)-1} f \circ \psi(v) \mathrm{d} v\right] \\
& =\frac{2^{(\alpha / k)-1}}{(b-a)^{(\alpha / k)}}\left[\int_{\psi^{-1}(a)}^{\psi^{-1}(a+b / 2)} d(\psi(v)-a)^{(\alpha / k)}(f(\psi(v)))\right] \\
& =\frac{1}{2} f\left(\frac{a+b}{2}\right)-\frac{1}{2} \int_{\psi^{-1}(a)}^{\psi^{-1}(a+b / 2)} f^{\prime}(\psi(v))\left(\frac{2(\psi(v))-a)}{b-a}\right)^{(\alpha / k)} \psi^{\prime}(v) \mathrm{d} v .
\end{aligned}
$$

By substituting $s=(2(\psi(v)-a) / b-a)$, we will get

$$
I_{2}=\frac{1}{2} f\left(\frac{a+b}{2}\right)-\frac{b-a}{4} \int_{0}^{1} f^{\prime}\left(a\left(\frac{2-s}{2}\right)+\frac{b s}{2}\right) s^{\alpha / k} \mathrm{~d} s \text {. }
$$

By summing (57) and (59), we get (54).

Remark 4. Under the assumption of Lemma 2, one can get the following results:

(i) If $k=1$ and $\psi$ is identity function in (54), then the identity (Lemma 3) stated in [2] is obtained.

(ii) If $k=1, \alpha=1$, and $\psi$ is identity function in (54), then the identity (Corollary 1) stated in [2] is obtained.

(iii) If $\psi$ is identity function in (54), then the identity (Lemma 3.1) stated in [36] is obtained.

Corollary 9. Under the assumption of Lemma 2 with $k=1$ in (54), the following identity holds:

$$
\begin{aligned}
& \frac{2^{\alpha-1} \Gamma(\alpha+1)}{(b-a)^{\alpha}}\left[I_{\psi^{-1}(a+b / 2)^{+}}^{\alpha, \psi}(f \circ \psi)\left(\psi^{-1}(b)\right)-I_{\psi^{-1}(a+b / 2)^{-}}^{\alpha, \psi}(f \circ \psi)\left(\psi^{-1}(a)\right)\right]-f\left(\frac{a+b}{2}\right) \\
& \quad=\frac{b-a}{4}\left[\int_{0}^{1} t^{\alpha} f^{\prime}\left(\frac{a t}{2}+\left(\frac{2-t}{2}\right) b\right) \mathrm{d} t+\int_{0}^{1} t^{\alpha} f^{\prime}\left(\left(\frac{2-t}{2}\right) a+\frac{b t}{2}\right) \mathrm{d} t\right] .
\end{aligned}
$$

Using above lemma, we give the following error bounds of the $k$-fractional Hadamard inequality.

Theorem 13. Let $f: I \longrightarrow \mathbb{R}$ be a differentiable mapping on $(a, b)$ with $a<b$. Also, suppose that $\left|f^{\prime}\right|^{q}$ is strongly convex function on $[a, b]$ with modulus $C \geq 0$ for $q \geq 1$, and $\psi$ is an increasing and positive monotone function on $(a, b]$, having $a$ continuous derivative $\psi^{\prime}(x)$ on $(a, b)$. Then, for $k>0$, the following $k$-fractional integral inequalities hold:

$$
\begin{aligned}
& \left|\frac{2^{(\alpha / k)-1} \Gamma_{k}(\alpha+k)}{(b-a)^{(\alpha / k)}}\left[{ }_{k} I_{\psi^{-1}(a+b / 2)^{+}}^{\alpha, \psi}(f \circ \psi)\left(\psi^{-1}(b)\right)+{ }_{k} I_{\psi^{-1}(a+b / 2)^{-}}^{\alpha, \psi}(f \circ \psi)\left(\psi^{-1}(a)\right)\right]-f\left(\frac{a+b}{2}\right)\right| \\
& \leq \frac{b-a}{4((\alpha / k)+1)}\left(\frac{1}{2((\alpha / k)+2)}\right)^{1 / q}\left[\left(\left(\frac{\alpha}{k}+1\right)\left|f^{\prime}(a)\right|^{q}+\left(\frac{\alpha}{k}+3\right)\left|f^{\prime}(b)\right|^{q}\right.\right. \\
& \left.\quad-\frac{C(b-a)^{2}((\alpha / k)+1)((\alpha / k)+4)}{2((\alpha / k)+3)}\right)^{1 / q}+\left(\left(\frac{\alpha}{k}+3\right)\left|f^{\prime}(a)\right|^{q}+\left(\frac{\alpha}{k}+1\right)\left|f^{\prime}(b)\right|^{q}\right. \\
& \left.\left.\quad-\frac{C(b-a)^{2}((\alpha / k)+1)((\alpha / k)+4)}{2((\alpha / k)+3)}\right)^{1 / q}\right]
\end{aligned}
$$


with $\alpha>0$.

Proof. From Lemma 2 and strongly convexity of $\left|f^{\prime}\right|$, let $q=1$, we have

$$
\begin{aligned}
& \left|\frac{2^{(\alpha / k)-1} \Gamma_{k}(\alpha+k)}{(b-a)^{(\alpha / k)}}\left[{ }_{k} I_{\psi^{-1}(a+b / 2)^{+}}^{\alpha,}(f \circ \psi)\left(\psi^{-1}(b)\right)+{ }_{k} I_{\psi^{-1}(a+b / 2)^{-}}^{\alpha, \psi}(f \circ \psi)\left(\psi^{-1}(a)\right)-f\left(\frac{a+b}{2}\right)\right]\right| \\
& \quad \leq \frac{b-a}{4}\left[\int_{0}^{1} t^{(\alpha / k)}\left|f^{\prime}\left(\frac{a t}{2}+\left(\frac{2-t}{2}\right) b\right)\right| \mathrm{d} t+\int_{0}^{1} t^{(\alpha / k)}\left|f^{\prime}\left(a\left(\frac{2-t}{2}\right)+\frac{b t}{2}\right)\right| \mathrm{d} t\right] \\
& \quad \leq \frac{b-a}{4}\left[\left(\left|f^{\prime}(a)\right|+\left|f^{\prime}(b)\right|\right) \int_{0}^{1} t^{(\alpha / k)} \mathrm{d} t-\frac{C}{2}(b-a)^{2} \int_{0}^{1} t^{(\alpha / k)+1}(2-t) \mathrm{d} t\right] \\
& \quad \leq \frac{b-a}{4((\alpha / k)+1)}\left[\left(\left|f^{\prime}(a)\right|+\left|f^{\prime}(b)\right|\right)-\frac{C(b-a)^{2}((\alpha / k)+4)((\alpha / k)+1)}{((\alpha / k)+2)((\alpha / k)+k)}\right] .
\end{aligned}
$$

Now, for $q>1$, we proceed as follows.

From Lemma 2 and using power mean inequality, we get

$$
\begin{aligned}
& \left|\frac{2^{(\alpha / k)-1} \Gamma_{k}(\alpha+k)}{(b-a)^{\alpha / k}}\left[{ }_{k} I_{\psi^{-1}(a+b / 2)^{+}}^{\alpha, \psi}(f \circ \psi)\left(\psi^{-1}(b)\right)+{ }_{k} I_{\psi^{-1}(a+b / 2)^{-}}^{\alpha, \psi}(f \circ \psi)\left(\psi^{-1}(a)\right)-f\left(\frac{a+b}{2}\right)\right]\right| \\
& \leq \frac{b-a}{4}\left(\int_{0}^{1} t^{(\alpha / k)} \mathrm{d} t\right)^{1-(1 / q)}\left[\left(\int_{0}^{1} t^{(\alpha / k)}\left|f^{\prime}\left(\frac{a t}{2}+\left(\frac{2-t}{2}\right) b\right)\right|^{q} \mathrm{~d} t\right)^{(1 / q)}\right. \\
& \left.\quad+\left(\int_{0}^{1} t^{(\alpha / k)}\left|f^{\prime}\left(\left(\frac{2-t}{2}\right) a+\frac{b t}{2}\right)\right|^{q} \mathrm{~d} t\right)^{(1 / q)}\right] .
\end{aligned}
$$

Strongly convexity of $\left|f^{\prime}\right|^{q}$ gives

$$
\begin{aligned}
& \left|\frac{2^{(\alpha / k)-1} \Gamma(\alpha+k)}{(b-a)}\left[{ }_{k} I_{\psi^{-1}(a+b / 2)^{+}}^{\alpha, \psi}(f \circ \psi)\left(\psi^{-1}(b)\right)+{ }_{k} I_{\psi^{-1}(a+b / 2)^{-}}^{\alpha, \psi}(f \circ \psi)\left(\psi^{-1}(a)\right)-f\left(\frac{a+b}{2}\right)\right]\right| \\
& \leq \frac{b-a}{4((\alpha / k)+1)^{1 / p}}\left[\left(\left|f^{\prime}(a)\right|^{q} \int_{0}^{1} \frac{t^{(\alpha / k)+1}}{2} \mathrm{~d} t+\left|f^{\prime}(b)\right|^{q} \int_{0}^{1}\left(\frac{2 t^{(\alpha / k)}-t^{(\alpha / k)+1}}{2}\right) \mathrm{d} t\right.\right. \\
& \left.\quad-\frac{C(b-a)^{2}}{4} \int_{0}^{1} t^{(\alpha / k)+1}(2-t) \mathrm{d} t\right)^{1 / q}+\left(\left|f^{\prime}(a)\right|^{q} \int_{0}^{1}\left(\frac{2 t^{(\alpha / k)}-t^{(\alpha / k)+1}}{2}\right) \mathrm{d} t\right. \\
& \left.\left.\quad+\left|f^{\prime}(b)\right|^{q} \int_{0}^{1} \frac{t^{(\alpha / k)+1}}{2} \mathrm{~d} t-\frac{C(b-a)^{2}}{4} \int_{0}^{1} t^{(\alpha / k)+1}(2-t) \mathrm{d} t\right)^{1 / q}\right] \\
& \leq \frac{b-a}{4((\alpha / k) 1)^{1 / p}}\left[\left(\frac{\left|f^{\prime}(a)\right|^{q}}{2((\alpha / k)+2)}+\frac{\left|f^{\prime}(b)\right|^{q}((\alpha / k)+3)}{2((\alpha / k)+1)((\alpha / k)+2)}-\frac{C(b-a)^{2}((\alpha / k)+4)}{4((\alpha / k)+2)((\alpha / k)+3)}\right)^{1 / q}\right. \\
& \left.\quad+\left(\frac{\left|f^{\prime}(a)\right|^{q}((\alpha / k)+3)}{2((\alpha / k)+1)((\alpha / k)+2)}+\frac{\left|f^{\prime}(b)\right|^{q}}{2((\alpha / k)+2)}-\frac{C(b-a)^{2}((\alpha / k)+4)}{4((\alpha / k)+2)((\alpha / k)+3)}\right)^{1 / q}\right]
\end{aligned}
$$


which after a little computation gives the required result.

Remark 5. Under the assumption of Theorem 13, one can get the following results:

(i) If $C=0$ and $\psi$ is identity function in (61), then the inequality (Theorem 3.1) stated in [36] is obtained. (ii) If $C=0, k=1$, and $\psi$ is identity function in (61), then the inequality (Theorem 5) stated in [2] is obtained.

(iii) If $q=1, C=0, k=1, \alpha=1$, and $\psi$ is identity function in (61), then the inequality (Theorem 2.2) stated in [43] is obtained.

Corollary 10. Under the assumption of Theorem 13 with $C=$ 0 in (61), the following inequality holds:

$$
\begin{aligned}
& \left|\frac{2^{(\alpha / k)-1} \Gamma_{k}(\alpha+k)}{(b-a)}\left[{ }_{k} I_{\psi^{-1}(a+b / 2)^{+}}^{\alpha, \psi}(f \circ \psi)\left(\psi^{-1}(b)\right)+{ }_{k} I_{\psi^{-1}(a+b / 2)^{-}}^{\alpha, \psi}(f \circ \psi)\left(\psi^{-1}(a)\right)\right]-f\left(\frac{a+b}{2}\right)\right| \\
& \leq \frac{b-a}{4((\alpha / k)+1)}\left(\frac{1}{2((\alpha / k)+2)}\right)^{1 / q}\left[\left(\left(\frac{\alpha}{k}+1\right)\left|f^{\prime}(a)\right|^{q}\right.\right. \\
& \left.\left.\quad+\left(\frac{\alpha}{k}+3\right)\left|f^{\prime}(b)\right|^{q}\right)^{1 / q}+\left(\left(\frac{\alpha}{k}+3\right)\left|f^{\prime}(a)\right|^{q}+\left(\frac{\alpha}{k}+1\right)\left|f^{\prime}(b)\right|^{q}\right)^{1 / q}\right] .
\end{aligned}
$$

Corollary 11. Under the assumption of Theorem 13 with $k=$

1 in (61), the following inequality holds:

$$
\begin{aligned}
& \left|\frac{2^{\alpha-1} \Gamma(\alpha+1)}{(b-a)^{\alpha}}\left[I_{\psi^{-1}(a+b / 2)^{+}}^{\alpha, \psi}(f \circ \psi)\left(\psi^{-1}(b)\right)+I_{\psi^{-1}(a+b / 2)^{-}}^{\alpha, \psi}(f \circ \psi)\left(\psi^{-1}(a)\right)\right]-f\left(\frac{a+b}{2}\right)\right| \\
& \quad \leq \frac{b-a}{4(\alpha+1)}\left(\frac{1}{2(\alpha+2)}\right)^{1 / q}\left[\left((\alpha+1)\left|f^{\prime}(a)\right|^{q}+(\alpha+3)\left|f^{\prime}(b)\right|^{q}\right.\right. \\
& \left.\quad-\frac{C(b-a)^{2}((\alpha / k)+1)(\alpha+4)}{2(\alpha+3)}\right)^{1 / q}+\left((\alpha+3)\left|f^{\prime}(a)\right|^{q}+(\alpha+1)\left|f^{\prime}(b)\right|^{q}\right. \\
& \left.\left.\quad-\frac{C(b-a)^{2}(\alpha+1)(\alpha+4)}{2(\alpha+3)}\right)^{1 / q}\right] .
\end{aligned}
$$

Corollary 12. Under the assumption of Theorem 13 with $\psi$ is identity function in (61), the following inequality holds:

$$
\begin{aligned}
& \left|\frac{2^{(\alpha / k)-1} \Gamma_{k}(\alpha+k)}{(b-a)^{(\alpha / k)}}\left[{ }_{k} I_{(a+b / 2)^{+}}^{\alpha} f(b)-{ }_{k} I_{(a+b / 2)^{-}}^{\alpha} f(a)\right]-f\left(\frac{a+b}{2}\right)\right| \\
& \leq \frac{b-a}{4((\alpha / k)+1)}\left(\frac{1}{2((\alpha / k)+2)}\right)^{1 / q}\left[\left(\left(\frac{\alpha}{k}+1\right)\left|f^{\prime}(a)\right|^{q}+\left(\frac{\alpha}{k}+3\right)\left|f^{\prime}(b)\right|^{q}\right.\right. \\
& \left.\left.\quad-\frac{C(b-a)^{2}((\alpha / k)+1)((\alpha / k)+4)}{2((\alpha / k)+3)}\right)^{1 / q}+\left(\left(\frac{\alpha}{k}+3\right)\left|f^{\prime}(a)\right|^{q}+\left(\frac{\alpha}{k}+1\right)\left|f^{\prime}(b)\right|^{q}-\frac{C(b-a)^{2}((\alpha / k)+1)((\alpha / k)+4)}{2((\alpha / k)+3)}\right)^{1 / q}\right] .
\end{aligned}
$$


Corollary 13. Under the assumption of Theorem 13 with $k=1, q=1, \alpha=1$, and $\psi$ as identity function in (61), the following inequality holds:

$$
\left|\frac{1}{b-a} \int_{a}^{b} f(v) \mathrm{d} v-f\left(\frac{a+b}{2}\right)\right| \leq \frac{b-a}{8}\left[\left|f^{\prime}(a)\right|+\left|f^{\prime}(b)\right|-\frac{5 C(b-a)^{2}}{12}\right] .
$$

Theorem 14. Let $f: I \longrightarrow \mathbb{R}$ be a differentiable mapping on $(a, b)$ with $a<b$. Also, suppose that $\left|f^{\prime}\right|^{q}$ is strongly convex function on $[a, b]$ for $q>1$, and $\psi$ is an increasing and positive monotone function on $(a, b]$, having a continuous derivative $\psi^{\prime}(x)$ on $(a, b)$. Then, for $k>0$, the following $k$-fractional integral inequalities hold:

$$
\begin{aligned}
& \left|\frac{2^{(\alpha / k)-1} \Gamma_{k}(\alpha+k)}{(b-a)^{(\alpha / k)}}\left[{ }_{k} I_{\psi^{-1}(a+b / 2)^{+}}^{\alpha, \psi}(f \circ \psi)\left(\psi^{-1}(b)\right)+{ }_{k} I_{\psi^{-1}(a+b / 2)^{-}}^{\alpha, \psi}(f \circ \psi)\left(\psi^{-1}(a)\right)\right]-f\left(\frac{a+b}{2}\right)\right| \\
& \quad \leq \frac{b-a}{16}\left(\frac{4}{(\alpha p / k)+1}\right)^{1 / p}\left[\left(\left(\left|f^{\prime}(a)\right|+3^{1 / q}\left|f^{\prime}(b)\right|\right)^{q}-\frac{2 C(b-a)^{2}}{3}\right)^{1 / q}\right. \\
& \left.\quad+\left(\left(3^{1 / q}\left|f^{\prime}(a)\right|+\left|f^{\prime}(b)\right|\right)^{q}-\frac{2 C(b-a)^{2}}{3}\right)^{1 / q}\right],
\end{aligned}
$$

with $\alpha>0$.

Proof. From Lemma 2 and using the property of modulus, we get

$$
\begin{aligned}
& \left|\frac{2^{(\alpha / k)-1} \Gamma_{k}(\alpha+k)}{(b-a)}\left[{ }_{k} I_{\psi^{-1}(a+b / 2)^{+}}^{\alpha, \psi}\left(f\left(\psi^{-1}(b)\right)\right)+{ }_{k} I_{\psi^{-1}(a+b / 2)^{-}}^{\alpha, \psi}(f \circ \psi)\left(\psi^{-1}(a)\right)-f\left(\frac{a+b}{2}\right)\right]\right| \\
& \quad \leq \frac{b-a}{4}\left[\int_{0}^{1}\left|t^{(\alpha / k)} f^{\prime}\left(\frac{t}{2} a+\left(\frac{2-t}{2}\right) b\right)\right| \mathrm{d} t+\int_{0}^{1}\left|t^{(\alpha / k)} f^{\prime}\left(\left(\frac{2-t}{2}\right) a+\frac{t}{2} b\right)\right| \mathrm{d} t\right] .
\end{aligned}
$$

Now applying Hölder's inequality for integrals, we get

$$
\begin{aligned}
& \left|\frac{2^{(\alpha / k)-1} \Gamma_{k}(\alpha+k)}{(b-a)^{(\alpha / k)}}\left[{ }_{k} I_{\psi^{-1}(a+b / 2)^{+}}^{\alpha, \psi}(f \circ \psi)\left(\psi^{-1}(b)\right)+{ }_{k} I_{g^{-1}(a+b / 2)^{-}}^{\alpha, \psi}(f \circ \psi)\left(\psi^{-1}(a)\right)-f\left(\frac{a+b}{2}\right)\right]\right| \\
& \quad \leq \frac{b-a}{4}\left[\left(\int_{0}^{1} t^{\alpha p / k} \mathrm{~d} t\right)^{1 / p}\left[\int_{0}^{1}\left|f^{\prime}\left(\frac{t}{2} a+\left(\frac{2-t}{2}\right) b\right)\right|^{q} \mathrm{~d} t\right]^{1 / q}\right. \\
& \left.\quad+\left(\int_{0}^{1} t^{\alpha p / k} \mathrm{~d} t\right)^{1 / p}\left[\int_{0}^{1}\left|f^{\prime}\left(\left(\frac{2-t}{2}\right) a+\frac{t}{2} b\right)\right|^{q} \mathrm{~d} t\right]^{1 / q}\right] .
\end{aligned}
$$


Using strongly convexity of $\left|f^{\prime}\right|^{q}$, we get

$$
\begin{aligned}
& \left|\frac{2^{(\alpha / k)-1} \Gamma_{k}(\alpha+k)}{(b-a)^{(\alpha / k)}}\left[{ }_{k} I_{\psi^{-1}(a+b / 2)^{+}}^{\alpha,}(f \circ \psi)\left(\psi^{-1}(b)\right)+{ }_{k} I_{\psi^{-1}(a+b / 2)^{-}}^{\alpha, a+b / 2}(f \circ \psi)\left(\psi^{-1}(a)\right)-f\left(\frac{a+b}{2}\right)\right]\right| \\
& \leq \frac{b-a}{4}\left(\frac{1}{(\alpha p / k)+1}\right)^{1 / p}\left[\left(\left|f^{\prime}(a)\right|^{q} \int_{0}^{1} \frac{t}{2} \mathrm{~d} t+\left|f^{\prime}(b)\right|^{q} \int_{0}^{1} \frac{2-t}{2} \mathrm{~d} t\right.\right. \\
& \left.\left.\quad-\frac{C(b-a)^{2}}{4} \int_{0}^{1} t(2-t) \mathrm{d} t\right)^{1 / q}+\left(\left|f^{\prime}(a)\right|^{q} \int_{0}^{1} \frac{2-t}{2} \mathrm{~d} t+\left|f^{\prime}(b)\right|^{q} \int_{0}^{1} \frac{t}{2} \mathrm{~d} t \frac{C(b-a)^{2}}{4} \int_{0}^{1} t(2-t) \mathrm{d} t\right)^{1 / q}\right] \\
& =\frac{b-a}{4}\left(\frac{1}{(\alpha p / k)+1}\right)^{1 / p}\left[\left(\left|f^{\prime}(a)\right|^{q}+3\left|f^{\prime}(b)\right|^{q}-\frac{2 C(b-a)^{2}}{3}\right)^{1 / q}\right. \\
& \left.\quad+\left(3\left|f^{\prime}(a)\right|^{q}+\left|f^{\prime}(b)\right|^{q}-\frac{2 C(b-a)^{2}}{3}\right)^{1 / q}\right] \\
& \leq
\end{aligned}
$$

Here, we have used the fact $a^{q}+b^{q} \leq(a+b)^{q}$, for $q>1$, $a, b \geq 0$. This completes the proof.

Remark 6. Under the assumption of Theorem 14, one can get the following results:

(i) If $C=0$ and $\psi$ is identity function in (69), then the inequality (Theorem 3.2) stated in [36] is obtained. (ii) If $k=1, C=0, \alpha=1$ and $\psi$ is identity function in (69), then the inequality (Theorem 2.3) stated in [43] is obtained.

Corollary 14. Under the assumption of Theorem 14 with $C=$ 0 in (69), the following inequality holds:

$$
\begin{aligned}
& \left|\frac{2^{(\alpha / k)-1} \Gamma_{k}(\alpha+k)}{(b-a)^{(\alpha / k)}}\left[{ }_{k} I_{\psi^{-1}(a+b / 2)^{+}}^{\alpha, \psi}(f \circ \psi)\left(\psi^{-1}(b)\right)+{ }_{k} I_{\psi^{-1}(a+b / 2)^{-}}^{\alpha, \psi}(f \circ \psi)\left(\psi^{-1}(a)\right)-f\left(\frac{a+b}{2}\right)\right]\right| \\
& \quad \leq \frac{b-a}{4}\left(\frac{4}{(\alpha p / k)+1}\right)^{1 / p}\left[\left|f^{\prime}(a)\right|+\left|f^{\prime}(b)\right|\right] .
\end{aligned}
$$

Corollary 15. Under the assumption of Theorem 14 with $k=$ 1 in (69), the following inequality holds:

$$
\begin{aligned}
& \left|\frac{2^{\alpha-1} \Gamma(\alpha+1)}{(b-a)^{\alpha}}\left[I_{\psi^{-1}(a+b / 2)^{+}}^{\alpha, \psi}(f \circ \psi)\left(\psi^{-1}(b)\right)+I_{\psi^{-1}(a+b / 2)^{-}}^{\alpha, \psi}\left(\psi^{-1}(a)\right)-f\left(\frac{a+b}{2}\right)\right]\right| \\
& \quad \leq \frac{b-a}{16}\left(\frac{4}{\alpha p+1}\right)^{1 / p}\left[\left(\left(\left|f^{\prime}(a)\right|+3^{1 / q}\left|f^{\prime}(b)\right|\right)^{q}-\frac{2 C(b-a)^{2}}{3}\right)^{1 / q}\right. \\
& \left.\quad+\left(\left(3^{1 / q}\left|f^{\prime}(a)\right|+\left|f^{\prime}(b)\right|\right)^{q}-\frac{2 C(b-a)^{2}}{3}\right)^{1 / q}\right] .
\end{aligned}
$$


Corollary 16. Under the assumption of Theorem 14 with $C=$ 0 and $k=1$ in (69), the following inequality holds:

$$
\begin{aligned}
& \left|\frac{2^{\alpha-1} \Gamma(\alpha+1)}{(b-a)^{\alpha}}\left[I_{\psi^{-1}(a+b / 2)^{+}}^{\alpha, \psi}(f \circ \psi)\left(\psi^{-1}(b)\right)+I_{\psi^{-1}(a+b / 2)^{-}}^{\alpha, \psi}(f \circ \psi)\left(\psi^{-1}(a)\right)-f\left(\frac{a+b}{2}\right)\right]\right| \\
& \quad \leq \frac{b-a}{4}\left(\frac{4}{\alpha p+1}\right)^{1 / p}\left[\left|f^{\prime}(a)\right|+\left|f^{\prime}(b)\right|\right] .
\end{aligned}
$$

Corollary 17. Under the assumption of Theorem 14 with $\psi$ is identity function in (69), the following inequality holds:

$$
\begin{aligned}
& \left|\frac{2^{(\alpha / k)-1} \Gamma_{k}(\alpha+k)}{(b-a)^{(\alpha / k)}}\left[{ }_{k} I_{(a+b / 2)^{+}}^{\alpha} f(b)+{ }_{k} I_{(a+b / 2)^{-}}^{\alpha} f(a)-f\left(\frac{a+b}{2}\right)\right]\right| \\
& \quad \leq \frac{b-a}{16}\left(\frac{4}{(\alpha p / k)+1}\right)^{1 / p}\left[\left(\left(\left|f^{\prime}(a)\right|+3^{1 / q}\left|f^{\prime}(b)\right|\right)^{q}-\frac{2 C(b-a)^{2}}{3}\right)^{1 / q}\right. \\
& \left.\quad+\left(\left(3^{1 / q}\left|f^{\prime}(a)\right|+\left|f^{\prime}(b)\right|\right)^{q}-\frac{2 C(b-a)^{2}}{3}\right)^{1 / q}\right] .
\end{aligned}
$$

Corollary 18. Under the assumption of Theorem 14 with $k=1, \alpha=1$, and $\psi$ is identity function in (69), the following inequality holds:

$$
\begin{aligned}
\left|\frac{1}{b-a} \int_{a}^{b} f(t) \mathrm{d} t-f\left(\frac{a+b}{2}\right)\right| \leq & \frac{b-a}{16}\left(\frac{4}{p+1}\right)^{1 / p}\left[\left(\left(\left|f^{\prime}(a)\right|+3^{1 / q}\left|f^{\prime}(b)\right|\right)^{q}-\frac{2 C(b-a)^{2}}{3}\right)^{1 / q}\right. \\
& \left.+\left(\left(3^{1 / q}\left|f^{\prime}(a)\right|+\left|f^{\prime}(b)\right|\right)^{q}-\frac{2 C(b-a)^{2}}{3}\right)^{1 / q}\right]
\end{aligned}
$$

\section{Conclusion}

In this paper, we have studied Hadamard inequalities and their error estimations using generalized Riemann-Liouville fractional integrals of strongly convex functions. The Hadamard inequalities obtained in this work are refinements as well as generalizations of many well-known inequalities. The error estimations of the Hadamard inequalities for differentiable strongly convex functions are better as compared to those which are obtained for convex functions. The authors are analyzing other well-known fractional integral operators for several kinds of functions in their future work.

\section{Data Availability}

No additional data are required.

\section{Conflicts of Interest}

The authors declare that they have no conflicts of interest.

\section{References}

[1] M. Z. Sarikaya, E. Set, H. Yaldiz, and N. Başak, "HermiteHadamard's inequalities for fractional integrals and related fractional inequalities," Mathematical and Computer Modelling, vol. 57, no. 9-10, pp. 2403-2407, 2013.

[2] M. Z. Sarikaya and H. S. Yildirim, "On Hermite-Hadamard type inequalities for Riemann-Liouville fractional integrals," Miskolc Mathematical Notes, vol. 17, no. 2, pp. 1049-1059, 2017.

[3] E. Set and I. Mumcu, "Hermite-Hadamard-Fejér type inequalities for conformable fractional integrals," Miskolc Mathematical Notes, vol. 20, no. 1, pp. 475-488, 2019. 
[4] G. Farid, "Hadamard and Fejér-Hadamard inequalities for generalized fractional integrals involving special functions," Konuralp Journal of Mathematics, vol. 4, no. 1, pp. 108-113, 2016.

[5] G. Rahman, T. Abdeljawad, F. Jarad, A. Khan, and K. S. Nisar, "Certain inequalities via generalized proportional Hadamard fractional integral operators," Advances in Difference Equations, vol. 2019, p. 454, 2019.

[6] H. Chen and U. N. Katugampola, "Hermite-Hadamard and Hermite-Hadamard-Fejér type inequalities for generalized fractional integrals," Journal of Mathematical Analysis and Applications, vol. 446, no. 2, pp. 1274-1291, 2017.

[7] J. Wang, X. Li, and C. Zhu, "Refinements of HermiteHadamard type inequalities involving fractional integrals," Bulletin of the Belgian Mathematical Society-Simon Stevin, vol. 20, no. 4, pp. 655-666, 2013.

[8] M. Avci, H. Kavurmaci, and M. Emin Raciila, "New inequalities of Hermite-Hadamard type via s-convex functions in the second sense with applications," Applied Mathematics and Computation, vol. 217, no. 12, pp. 5171-5176, 2011.

[9] N. Mehreen and M. Anwar, "Hermite-Hadamard and Hermite-Hadamard-Fejer type inequalities for p-convex functions via new fractional conformable integral operators," Journal of Mathematics and Computer Science, vol. 19, no. 4, pp. 230-240, 2019.

[10] P. O. Mohammed, M. Z. Sarikaya, and D. Baleanu, "On the generalized Hermite-Hadamard inequalities via the tempered fractional integrals," Symmetry, vol. 12, no. 4, p. 595, 2020.

[11] S. Mubeen, S. Iqbal, and M. Tomar, "On Hermite-Hadamard type inequalities via fractional integrals of a function with respect to another function and $k$-parameter," Journal of Inequalities and Applications, vol. 10, p. 5, 2016.

[12] K. Oldham and J. Spanier, The Fractional Calculus, Academic Press, New York, NY, USA, 1974.

[13] K. S. Miller and B. Ross, An Introduction to the Fractional Calculus and Fractional Differential Equations, John Wiley \& Sons, New York, NY, USA, 1993.

[14] S. Das, "Introduction to fractional calculus," in Functional Fractional Calculus, Springer, Berlin, Heidelberg, 2011.

[15] K. S. Nisar, D. Baleanu, and M. M. Al Qurashi, "Fractional calculus and application of generalized Struve function," Springerplus, vol. 5, no. 1, p. 910, 2016.

[16] K. S. Nisar, G. Rahman, J. Choi, S. Mubeen, and M. Arshad, "Generalized hypergeometric k-functions via $(k, s)$-fractional calculus," The Journal of Nonlinear Sciences and Applications, vol. 10, no. 4, pp. 1791-1800, 2017.

[17] P. Agarwal, M. Chand, J. Choi, and G. Singh, "Certain fractional integrals and image formulas of generalized- $k$ Bessel function," Communications of the Korean Mathematical Society, vol. 33, no. 2, pp. 423-436, 2018.

[18] D. Baleanu and G. C. Wu, "Some further results of the Laplace transform for variable-order fractional difference equations," Fractional Calculus and Applied Analysis, vol. 22, no. 6, pp. 1641-1654, 2019.

[19] D. Baleanu, S. Rezapour, and Z. Saberpour, "On fractional integro-differential inclusions via the extended fractional Caputo-Fabrizio derivation, bound," Boundary Value Problems, vol. 2019, no. 1, p. 79, 2019.

[20] G. Singh, P. Agarwal, S. Araci, and M. Acikgoz, "Certain fractional calculus formulas involving extended generalized Mathieu series," Advances in Difference Equations, vol. 2018, p. 144, 2018.

[21] A. A. El-Sayed and P. Agarwal, "Numerical solution of multiterm variable-order fractional differential equations via shifted Legendre polynomials," Mathematical Methods in the Applied Sciences, vol. 42, no. 2, pp. 3978-3991, 2019.

[22] H. Khalil, R. A. Khan, D. Baleanu, and M. M. Rashidi, "Some new operational matrices and its application to fractional order Poisson equations with integral type boundary constrains," Computers \& Mathematics with Applications, vol. 78, no. 6, pp. 1826-1837, 2019.

[23] K. S. Nisar, G. Rahman, and K. Mehrez, "Chebyshev type inequalities via generalized fractional conformable integrals," Journal of Inequalities and Applications, vol. 2019, p. 245, 2019.

[24] M. U. Farooq, M. S. Khan, M. S. Khan, and A. Hajizadeh, "Flow of viscous fluid over an infinite plate with CaputoFabrizio derivatives," Open Journal of Mathematical Sciences, vol. 3, no. 1, pp. 115-120, 2019.

[25] V. V. Kulish and J. L. Lage, "Application of fractional calculus to fluid mechanics," Journal of Fluids Engineering, vol. 124, no. 3, pp. 803-806, 2002.

[26] Z. Odibat and S. Momani, "The variational iteration method: an efficient scheme for handling fractional partial differential equations in fluid mechanics," Computational and Applied Mathematics, vol. 58, no. 11-12, pp. 2199-2208, 2009.

[27] C. N. Angstmann, B. I. Henry, and A. V. McGann, "A fractional-order interactivity and recovery SIR model," Fractal and Fractional, vol. 1, no. 1, p. 11, 2017.

[28] A. Akgül, A. Kilicman, and D. Baleanu, "A new approach for one-dimensional sine-Gordon equation," Advances in Difference Equations, vol. 2016, no. 8, p. 8, 2016.

[29] B. T. Polyak, "Existence theorems and convergence of minimizing sequences in extremum problems with restrictions," Soviet Mathematics. Doklady, vol. 7, pp. 72-75, 1966.

[30] J. P. Vial, "Strong convexity of sets and functions," Journal of Mathematical Economics, vol. 9, no. 1-2, pp. 187-205, 1982.

[31] M. U. Awan, M. A. Noor, T.-S. Du, and K. I. Noor, "New refinements of fractional Hermite-Hadamard inequality," Revista de la Real Academia de Ciencias Exactas, Físicas y Naturales. Serie A. Matemáticas, vol. 113, no. 1, pp. 21-29, 2019.

[32] F. Zhang, G. Farid, and S. B. Akbar, "Inequalities for Riemann-Liouville fractional integrals of strongly $(s, m)$-convex functions," Journal of Mathematics, vol. 2021, Article ID 5577203, 14 pages, 2021.

[33] S. Mubeen and G. M. Habibullah, " $k$ Fractional integrals and applications," International Journal of Contemporary Mathematical Sciences, vol. 7, pp. 89-94, 2012.

[34] S. Mubeen and A. Rehman, "A note on $k$-Gamma function and Pochhammer $k$-symbol," Journal of Mathematical Sciences, vol. 6, no. 2, pp. 93-107, 2014.

[35] G. Farid, A. U. Rehman, and M. Zahra, "On Hadamard-type inequalities for $k$-fractional integrals," Nonlinear Functional Analysis and Applications, vol. 21, no. 3, pp. 463-478, 2016.

[36] G. Farid, A. U. Rehman, and M. Zahra, "On Hadamard inequalities for $k$-fractional integrals," Konuralp Journal of Mathematics, vol. 4, no. 2, pp. 79-86, 2016.

[37] A. A. Kilbas, H. M. Srivastava, and J. J. Trujillo, Theory and Applications of Fractional Differential Equations, NorthHolland Math. Stud., Elsevier, New York, NY, USA, 2006.

[38] Y. C. Kwun, G. Farid, W. Nazeer, S. Ullah, and S. M. Kang, "Generalized Riemann-Liouville $k$-fractional integrals associated with ostrowski type inequalities and error bounds of hadamard inequalities," IEEE Access, vol. 6, pp. 64946-64953, 2018. 
[39] S. G. Samko, A. A. Kilbas, and O. I. Marichev, Fractional Integrals and Derivatives, Theory and Applications, Gordon and Breach Science Publishers, London, UK, 1993.

[40] K. Liu, J. Wang, and D. O'regan, “On the Hermite-Hadamard type inequality for $\psi$-Riemann-Liouville fractional integrals via convex functions," Journal of Inequalities and Applications, vol. 2019, p. 27, 2019.

[41] M. U. Awan, S. Talib, Y. M. Chu, M. A. Noor, and K. I. Noor, "Some new refinements of Hermite-Hadamard-type inequalities involving $\psi_{k}$-Riemann-Liouville fractional integrals and applications," Journal of Mathematical Problems in Engineering, vol. 2020, no. 1, pp. 1-10, 2020.

[42] S. S. Dragomir and R. P. Agarwal, "Two inequalities for differentiable mappings and applications to special means of real numbers and to trapezoidal formula," Applied Mathematics Letters, vol. 11, no. 5, pp. 91-95, 1998.

[43] U. S. Kirmaci, "Inequalities for differentiable mappings and applications to special means of real numbers and to midpoint formula," Applied Mathematics and Computation, vol. 147, no. 1, pp. 137-146, 2004.

[44] N. Merentes and K. Nikodem, "Remarks on strongly convex functions," Aequationes Mathematicae, vol. 80, no. 1-2, pp. 193-199, 2010. 